standard FNA needle. We hereby present data on yield of 20G Cook EchoTip Procore ${ }^{\mathrm{TM}}$ FNB needle.

Aim To compare the diagnostic yield of 20G FNB needle and compare the incremental yield of histological over cytological preparation of tissue acquired.

Methodology Prospective non-blinded randomised study. All patients undergoing EUS guided FNA/FNB from January 2016 to January 2018, for all upper GI and HPB lesions were included. FNB was performed using Cook Echotip Procore 20GTM needle, Olympus EU-ME2TM processor and linear echoendoscopes was used. All patients had 2 or 4 passes done with the same needle, specimens from each pass were randomly collected in BD Cytorich $^{\mathrm{TM}}$ or Formalin. Each preservative had either 1 or 2 passes of material. Only C5 malignancy diagnosis from the primary pathologist or from second expert opinion with MDT acceptance was considered positive for cancer, other non-malignant conditions was considered as positive for statistical purposes only when clear diagnosis was offered by pathologist.

Results In total, 147 patients had EUS guided sampling and 111 patients had EUS FNB with 20G FNB needle. $68 \mathrm{M}: 43$ F. Average age was 66.7 years $( \pm 11.3)$. Of these, 102 had samples both for cytology and histology. Final diagnosis was made in $92(90.2 \%)$ patients. Pancreatic cancer was seen in $52(51 \%)$ patients, cholangiocarcinoma in 7 (6.7\%), NET/ GIST in $11(10.8 \%)$, Lymphoma in 11 (10.8\%), malignant nodes in 6 (5.9\%), other cancers (e.g. rhabdomyosarcoma) in 6 (5.9\%), benign (e.g. chronic pancreatitis, autoimmune pancreatitis, paraganglioma, reactive nodes etc) in 8 (7.7\%), and no diagnosis was made in 10 (9.8\%).

Histological processing provided answers in 87 (78.3\%) patients with combined cytological and histological processing providing diagnosis in the rest of 15 (13.5\%) with overall diagnostic yield of $91.9 \%$ (102 patients).

Conclusion Our study results show that adequate samples can be obtained for histological processing with Cooke Procor$\mathrm{e}^{\mathrm{TM}}$ FNB needles with good cumulative diagnostic yield (92\%) and significant diagnostic yield from preferentially processing samples for histological assessment rather than standard cytology, possibly due to better preservation of tissue architecture and cell morphology. Limitations include non-specialist reporting cytopathologist and non-randomised retrospective design.

\section{PTH-042 COLORECTAL STENTING AS A BRIDGE TO SURGERY: A DECADE OF SINGLE CENTRE SUCCESS}

Lindsay Steward*, Miriam Manook, Waveney Stafford, Fiona Taylor, Stefano Andreani, Alan Watson. Queen's Hospital, Romford, Little Berkhamsted, UK

\subsection{6/gutjnl-2018-BSGAbstracts.63}

Introduction Colonic stenting in obstructing left sided colorectal cancer (CRC) using self-expanding metallic stents has been utilised as a conservative management intervention, as well as a bridge to definitive surgery. Despite high reported success rates technically and clinically of stent deployment, as well as improved rates of primary anastomosis, controversy remains about the use of this procedure as a bridge to surgery. We report on a decade of single centre experience.

Methods Retrospective analysis of patients requiring urgent colorectal cancer stenting as a bridge to surgery between 2006 and 2017. Primary outcomes measured were clinical and technical success. Secondary outcomes recorded were survival, complications, primary anastomosis and stoma formation

Results 36 patients underwent CRC stenting with the intention of being a bridge to elective surgery, of whom $44 \%$ were female. Mean age was 65.7 years. The majority of cases $(84 \%)$ were undertaken by one of the 3 main operators. At staging, 9 patients (25\%) were T3; 17 (47.3\%) T4. 25\% of patients were ASA 3. Technical success was reported in $89 \%$ of cases and clinical success $86 \%$. At elective surgery primary anastomosis was achieved in $61 \%$. 5 reported early complications, including 2 clinical perforations and 2 radiological perforations. Mean survival post stenting procedure was 24 months (range 3-55 months), with death due to metastatic CRC. There were no deaths within 30 days.

Conclusions We demonstrate excellent technical success with this treatment modality. By temporising patients using endoscopic stenting, patients are optimised for elective surgery by colorectal surgeons with good primary anastomosis rates, and excellent post procedure mortality.

\section{PTH-043 ADULT COELIAC DISEASE REMISSION ASSESSMENT: DOES A D1 BIOPSY INCREASE THE DETECTION OF VILLOUS ATROPHY?}

${ }^{1}$ Lauren Marks*, ${ }^{1}$ Michelle Lau, 'Matthew Kurien, ${ }^{2}$ Marios Hadjivassiliou, 'Peter Mooney, ${ }^{3}$ ProfSimon Cross, ${ }^{1}$ David Sanders. ${ }^{1}$ Academic Unit of Gastroenterology, Royal Hallamshire Hospital, Sheffield, UK; ${ }^{2}$ Department of Neurology, Royal Hallamshire Hospital, Sheffield, UK; ${ }^{3}$ Department of Pathology, Royal Hallamshire Hospital, Sheffield, UK

\subsection{6/gutjnl-2018-BSGAbstracts.64}

Introduction The duodenal bulb (D1) has been shown to be a sensitive site for detecting villous atrophy (VA) in newly diagnosed coeliac disease (CD), however there is a scarcity of data from those with established CD. In patients with established $\mathrm{CD}$, we aim to establish whether D1 biopsies improved the identification of VA compared to biopsies from the second part of the duodenum (D2) alone.

Methods 251 patients with established CD were prospectively recruited from the endoscopy department at the Royal Hallamshire Hospital between 2013 and 2017. These patients were undergoing repeat gastroscopy to assess dietary adherence. All patients underwent a gastroscopy, with one biopsy taken from the duodenal bulb and four from D2. Biopsies were classified according to Marsh criteria. We assessed concordance of histology between the D1 and D2 sites, and 95\% confidence intervals were calculated for all results using a binominal distribution.

Results 251 patients were recruited $(70.5 \%$ female, age range 17-81 years, median age 53 years) having been on a glutenfree diet for a median duration of 6 years. Concordant results: $35.1 \%$ ( $n=88,95 \% \mathrm{CI}: 29.16$ to 40.96$)$ had normal duodenal biopsies in both D1 and D2; 32.3\% ( $\mathrm{n}=81,95 \%$ CI: 26.49 to 38.05$)$ had VA in D1 and D2; $18.3 \%(n=46$, 95\% CI: 13.54 to 23.11 ) had raised intra-epithelial lymphocytes (IELs) only in both D1 and D2. Disconcordant results: $4.4 \%(\mathrm{n}=11,95 \% \mathrm{CI}: 1.85$ to 6.91$)$ had VA in D1 but not D2; $2.4 \%(n=6,95 \% \mathrm{CI}: 0.50$ to 4.28$)$ had raised IELs in D1 but normal histology in D2. $2.8 \%(n=7,95 \%$ CI: 0.75 to 4.83) had VA in D2 but normal histology in D1; $4.8 \%$ $(\mathrm{n}=12,95 \% \mathrm{CI}: 2.14$ to 7.42$)$ had IELs in D2 but normal histology in D1.

Conclusions VA was confined to the duodenal bulb in $4.4 \%$ of patients with established CD. Thus a D1 biopsy in addition 